\title{
Multimodal Preventive Trial for Alzheimer's Disease: MIND-ADmini Pilot Trial Study Design and Progress
}

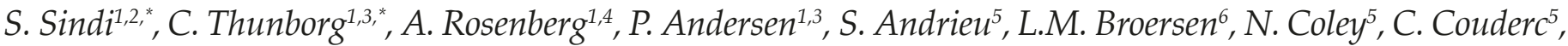 \\ C.Z. Duval, ${ }^{7,8}$, G. Faxen-Irving'1, G. Hagman ${ }^{3}$, M. Hallikainen ${ }^{4}$, K. Håkansson ${ }^{1,3}$, J. Lehtisalo ${ }^{4,9}$, N. Levak ${ }^{1,3}$, \\ F. Mangialasche ${ }^{1}$, J. Pantel ${ }^{7,8}$, E. Kekkonen ${ }^{4}$, A. Rydström ${ }^{1}$, A. Stigsdotter-Neely ${ }^{10,11}$, A. Wimo ${ }^{1,12}$, T. Ngandu ${ }^{8}$, \\ H. Soininen ${ }^{4}$, T. Hartmann $n^{7,13}$, A. Solomon ${ }^{1,2,4,}{ }^{* *}$, M. Kivipelto ${ }^{1,2,3,4,14,{ }^{* *}}$

\begin{abstract}
1. Division of Clinical Geriatrics, Department of Neurobiology, Care Sciences and Society, Karolinska Institute, Sweden; 2. The Ageing Epidemiology (AGE) Research Unit, School of Public Health, Imperial College London, London, United Kingdom; 3. Theme Inflammation and Aging, Karolinska University Hospital, Stockholm, Sweden; 4. Department of Neurology, Institute of Clinical Medicine, University of Eastern Finland, Kuopio, Finland; 5. Department of Clinical Epidemiology and Public health, CHU de Toulouse, and INSERM, University of Toulouse UMR1027, Toulouse, France; 6. Danone Nutricia Research, Advanced Medical Nutrition, Utrecht, Netherland; 7. German Institute for Dementia Prevention (DIDP), Saarland University, Homburg, Germany; 8. Institute of General Practice, Goethe University Frankfurt a.M., Germany; 9. Population Health Unit, Department of Public Health and Welfare, Finnish Institute for Health and Welfare, Helsinki, Finland; 10. Department of Social and Psychological Studies, Karlstad University, Karlstad, Sweden; 11. Engineering Psychology, Luleå University of Technology, Luleå, Sweden; 12. Centre for Research \& Development, Uppsala University/County Council of Gävleborg, Gävle, Sweden; 13. Department of Experimental Neurology, Medical Faculty, Saarland University, Homburg, Germany; 14. Stockholms Sjukhem, Research \& Development Unit, Stockholm, Sweden; *these authors contributed equally; ${ }^{* *}$ these senior authors contributed equally
\end{abstract}

Corresponding Author: Professor Miia Kivipelto, MD, PhD, Karolinska Institutet, Dept NVS, Division of Clinical Geriatrics, Center for Alzheimer Research QA32, Karolinska vägen 37 A, SE-171 64 Solna, Sweden; Mobile: +46 (0)73 99409 22, email: miia.kivipelto@ki.se

\begin{abstract}
BACKGROUND: Interventions simultaneously targeting multiple risk factors and mechanisms are most likely to be effective in preventing cognitive impairment. This was indicated in the Finnish Geriatric Intervention Study to Prevent Cognitive Impairment and Disability (FINGER) testing a multidomain lifestyle intervention among at-risk individuals. The importance of medical food at the early symptomatic disease stage, prodromal Alzheimer's disease (AD), was emphasized in the LipiDiDiet trial. The feasibility and effects of multimodal interventions in prodromal AD are unclear.
\end{abstract}

OBJECTIVES: To evaluate the feasibility of an adapted FINGERbased multimodal lifestyle intervention, with or without medical food, among individuals with prodromal AD.

METHODS: MIND-ADmini is a multinational proof-of-concept 6-month randomized controlled trial (RCT), with four trial sites (Sweden, Finland, Germany, France). The trial targeted individuals with prodromal AD defined using the International Working Group-1 criteria, and with vascular or lifestyle-related risk factors. The parallel-group RCT includes three arms: 1) multimodal lifestyle intervention (nutritional guidance, exercise, cognitive training, vascular/metabolic risk management and social stimulation); 2) multimodal lifestyle intervention+medical food (Fortasyn Connect); and 3) regular health advice/ care (control group). Primary outcomes are feasibility and adherence. Secondary outcomes are adherence to the individual intervention domains and healthy lifestyle changes.

RESULTS: Screening began on 28 September 2017 and was completed on 21 May 2019. Altogether 93 participants were randomized and enrolled. The intervention proceeded as planned.

CONCLUSIONS: For the first time, this pilot trial tests the feasibility and adherence to a multimodal lifestyle intervention, alone or combined with medical food, among individuals with prodromal AD. It can serve as a model for combination therapy trials (non-pharma, nutrition-based and/or pharmacological interventions).
Key words: Dementia, Alzheimer's disease, prevention, lifestyle, intervention, multimodal, randomized controlled trial.

\section{Introduction}

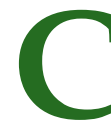
urative or disease modifying treatments for dementia and Alzheimer's disease (AD) are currently not widely available, and prevention has been identified as a global priority. Estimates have shown that at least $40 \%$ of dementia cases are attributable to modifiable vascular and lifestyle-related risk factors (e.g. hypertension, diabetes mellitus, smoking, obesity, physical inactivity, depression, low education), creating a clear window of opportunity for prevention (1). These are consistent with the recent World Health Organization (WHO) Guidelines for risk reduction of cognitive decline and dementia (2). Several randomized controlled trials (RCTs) have tested unimodal interventions for AD/ dementia prevention (e.g. a single drug or single lifestyle intervention, such as cognitive training or physical activity), with negative or very modest results (3).

Recent clinic-neuropathological studies have demonstrated that only $10-30 \%$ of clinical AD cases have pure $\mathrm{AD}$ pathology, and vascular pathology is the most common comorbidity (4). Thus, unimodal approaches targeting a single pathology are probably insufficient to prevent dementia. Given the multifactorial etiology of late-onset $\mathrm{AD}$, multimodal interventions simultaneously targeting multiple risk factors and disease mechanisms are more likely to be effective. Some recent large-scale European RCTs have tested multimodal preventive interventions in community-dwelling older adults (5). The 2-year Finnish Geriatric Intervention Study to 
Prevent Cognitive Impairment and Disability (FINGER) (6) (including nutritional guidance, exercise, cognitive training, vascular/metabolic risk management and social stimulation) showed significant benefits on cognition, physical function and health-related quality of life among older adults from the general population, who had an increased risk for dementia (6). In the FINGER trial, at-risk participants were selected using a validated dementia risk score (Cardiovascular Risk Factors, Aging and Dementia - CAIDE Dementia Risk Score) (7). Results from two other multimodal lifestyle trials have also been published; the 6-year Dutch Prevention of Dementia by Intensive Vascular Care (PreDIVA) trial (8) and the 3-year French Multidomain Alzheimer Preventive Trial (MAPT) (9). While these interventions did not have significant effects on the primary outcome, post-hoc analyses suggested potential benefit among individuals with higher dementia risk (in MAPT individuals with a CAIDE score of at least 6 and those with amyloid pathology; in PreDIVA individuals with untreated hypertension). Taken together, these findings further emphasize the importance of targeting individuals with elevated dementia risk (10), and the need for better-defined at-risk groups to achieve an optimal intervention response.

Although patients with prodromal AD $(11,12)$ represent a group with high risk of progressing to dementia, no effective treatment options are widely available for these individuals. Multimodal lifestylebased interventions have not been tested in this population. However, the European LipiDiDiet trial (13) investigated the effects of a medical food containing Fortasyn Connect, a combination of nutrients developed to ameliorate synaptic dysfunction. LipiDiDiet was the first multinational RCT to target individuals with prodromal AD identified with the International Working Group 1 (IWG-1) criteria (12). The 2-year intervention did not show significant effects on the primary outcome, i.e. change in cognitive performance measured with a Neuropsychological Test Battery (NTB) composite score, but there were significant beneficial effects on cognitive-functional performance and brain structure (i.e., less cognitive-functional decline and reduction in hippocampal volume (key secondary outcome)) in the intervention group. Longer-term (3-year) intervention benefits were reported on the primary cognitive outcome, and most secondary outcomes, including cognitivefunctional and brain atrophy measures (14), which supported beneficial effects of this nutrient combination.

The rationale for combining a lifestyle multimodal intervention with medical food is based on evidence indicating synergistic effects between different intervention components (e.g. omega-3 fatty acids and physical activity) (15-18). This concept has been recently tested in the MAPT trial, where the combined effects of the multidomain lifestyle intervention with omega- 3 fatty acid supplementation were assessed among individuals at higher risk for dementia, and led to improved memory score and cognitive status, compared to the placebo
(19). Further, nutrient deficiencies in prodromal AD are common (20), and medical food may complement dietary guidance for optimal effects. Building on these results, the Multimodal Preventive Trial for Alzheimer's Disease (MIND-ADmini) pilot trial was designed as a proof-ofconcept trial to assess the feasibility of a multimodal lifestyle intervention, with and without medical food, among individuals with prodromal AD (12).

\section{Materials and methods}

\section{Study design}

The MIND-ADmini (ClinicalTrials.gov identifier NCT03249688) is a 6-month multinational (Sweden, Finland, Germany, and France) proof-of-concept RCT with three parallel groups: 1) multimodal lifestyle intervention; 2) multimodal lifestyle intervention + medical food; and 3) regular health advice/care (control). The trial does not include an arm with medical food alone, as this was investigated in the LipiDiDiet trial (13). Participants were randomized in 1:1:1 ratio in blocks of six (computer generated allocation, two individuals randomly allocated to each group) at each of the four sites after screening by the study nurse. Outcome evaluators were blinded to the randomization group and were not involved in intervention activities. Similar to the FINGER trial, group allocation was not actively disclosed to the participants, and participants were instructed not to discuss the intervention with the outcome evaluators. The study design is summarized in Figure 1. Intervention duration for the core trial was 6 months, and a 6-month optional extension was conducted at the Swedish site.

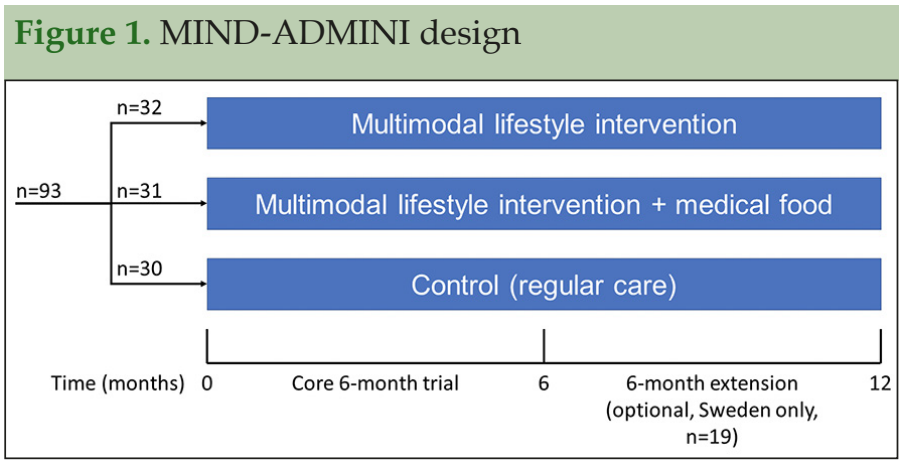

\section{Participants}

\section{Inclusion and exclusion criteria}

Screening began on 28 September 2017 and was completed on 21 May 2019. Participants were recruited from memory clinics in Stockholm, Sweden and Toulouse, France; via local media advertisement in Frankfurt Germany; and via the university hospital neurology clinic and previous research cohorts in Kuopio, Finland (i.e., 
Table 1. MIND-AD trial inclusion criteria

- Age 60-85 years.

- Mini-Mental State Examination (MMSE) (21) score of $\geq 24$

- Availability of a study partner

Criteria for episodic memory disorder defined as -1 SD on 2 out of 8 tests, at least 1 memory

\section{Memory}

FCSRT - delayed free recall $\leq 8$

FCSRT free recall - learning $\leq 22$

WMS-R story delayed recall $(\%) \leq 75 \%$

WMS-R delayed recall of figures $(\%) \leq 75 \%$

\section{Non-memory}

TMT $\mathrm{A} \geq 60$

TMT $\mathrm{B} \geq 150$

Symbol Digit Substitution Test $\leq 35$ (120 sec.)

Category Fluency $\leq 16$ (60 sec.)

Criteria for underlying AD pathology within 1 year prior to screening by either

CSF $\beta$-amyloid (1-42/1-40) *10 ratio $<1$ and/or total-tau and/or phospho-tau and / or $\beta$-amyloid 42 based on local cut-offs OR

MRI evidence for medial temporal lobe atrophy (MTA score 1 or higher) OR

Abnormal FDG PET and/or PiB PET compatible with AD type changes

Lifestyle index: Score of 2 or above required for inclusion. (Each 'yes' answer is given one point)

- Physical activity less than 2.5 hours a week* (defined as physical activity intensive enough to lead to sweating and some breathlessness)

- Diet - less than 5 portions* of fruits and vegetables per day

- Diet - less than 2 portions* of fish per week

- Hypertension (diagnosed by physician or current antihypertensive treatment or $\mathrm{SBP}>140 \mathrm{mmHg}$ or $\mathrm{DBP}>90 \mathrm{mmHg}$ )

- Diabetes (type 1 or 2 diagnosed by physician; or current diabetes medication; or recorded elevated fasting blood glucose or $\mathrm{HbA} 1 \mathrm{C}$ as per local guidelines within the past 6 months)

- Sleep disturbances, depressive symptoms or psychological stress symptoms, for at least one month, judged by the clinician as having some impact on everyday life.

*Based on WHO and national guidelines; AD: Alzheimer's disease; CSF: cerebrospinal fluid; DSB: diastolic blood pressure; FCSRT: Free and Cued Selective Reminding Test; FDG PET: fluorodeoxyglucose (FDG)-positron emission tomography (PET); MTA: medial temporal lobe atrophy; PiB PET: Pittsburgh compound B positron emission tomography; SBP: Systolic blood pressure; TMT: Trail making test; WMS-R: Wechsler Memory Scale-revised.

participants who had previously participated in studies and given their consent to be re-contacted for other studies). Inclusion criteria are listed in Table 1.

The IWG-1 criteria were used for prodromal AD, defined as having episodic memory impairment and evidence for underlying AD pathology (12). Episodic memory disorder was defined as -1 SD on at least 2 out of 8 tests, of which at least 1 is a memory test (Table 1). AD pathology was defined as having at least one abnormal cerebrospinal fluid (CSF) or neuroimaging biomarker, both similar to the LipiDiDiet trial (13) (Table 1).

A lifestyle index was used for screening to identify individuals with multiple modifiable vascular or lifestylerelated risk factors and thus potential for improvement. The score was calculated by adding one point for each risk factor (see Table 1). For the last item 'sleep disturbances, depressive symptoms, or psychological stress symptoms', having one of these three items was sufficient to receive a score of one point. A total of two points or more were required for inclusion.

Exclusion criteria were: conditions affecting safe engagement in the intervention (i.e., the exercise training program), Dementia according to Diagnostic and Statistical Manual of Mental Disorders, Fourth
Edition (DSM-IV), use of omega-3 preparations (> 500mg EPA+DHA per day), alcohol or drug abuse, concomitant severe disease (e.g., recent history of myocardial infarction or cancer), major depressive disorders (DSMIV), intake of supplements for vitamin B6, B12, folic acid, vitamin $C$ and/or $E>200 \%$ of the recommended daily intake unless prescribed by physician, subjects with MRI (or CT) scan consistent with a diagnosis of stroke, intracranial bleeding, mass lesion or normal pressure hydrocephalus. Individuals with an MRI scan demonstrating minimal white matter changes (Fazekas scale for white matter lesions $<=3$ ) and up to 1-2 lacunar infarcts that were judged to be clinically insignificant were allowed for inclusion. Severe loss of vision or communicative ability, conditions preventing cooperation as judged by the study physician, concomitant participation in any intervention trial (in the last 30 days) were also exclusion criteria.

\section{Intervention program}

The multimodal intervention program in MINDADmini is based on the FINGER protocol (6), and was adapted for participants with prodromal AD. The 
Table 2. FINGER protocol adaptations made in the delivery, content and evaluation of the intervention program in MIND-ADMINI pilot trial

- Physical exercise sessions were performed in small groups of 4-6 participants.

- Physical exercise training program was conducted when the gym facilities were not occupied by other customers.

- The components of the physical exercise program was performed in 3-week bouts (i.e., 3-w aerobic and 3-w anaerobic) to facilitate adherence/ motivation.

- Physical activity was evaluated with actigraphy for objective measures and to minimize demands of handling exercise diaries.

- The content of the cognitive training program was adapted to the cognitive functioning level of participants with prodromal AD.

- Cognitive training sessions were carried out on site when possible to facilitate adherence.

- Delivery of diet counseling was made in cooperation with study partners to increase adherence when feasible.

- Support for transport to study center was provided if the participant had orientation problems in new areas.

FINGER protocol adaptations (Table 2) were made in order to better tailor the intervention to this target population, e.g. adapting the lifestyle intervention schedule to reduce the burden of frequent travel to the trial sites.

At baseline, the study nurse gave all participants oral and written information and advice on healthy diet and physical, cognitive, and social activities that are beneficial for the management of vascular risk factors and disability prevention. Blood samples were collected from all participants twice during the study, at baseline and 6 months (and at the 12 months visit in the extension in Sweden). In case of clinically relevant abnormal findings from blood results, participants were provided with information and advice to contact primary health care or a referral to primary health care. All participants had a chance to contact the study nurse by telephone or e-mail when needed.

\section{The multimodal lifestyle intervention}

In addition to general health advice, participants in the two intervention arms (lifestyle and lifestyle+medical food) received the multimodal lifestyle program. The multimodal lifestyle program consisted of: (1) Nutritional guidance; (2) Physical exercise; (3) Cognitive training; (4) Intensive monitoring and management of vascular and metabolic risk factors; (5) Social stimulation. The different intervention components were introduced gradually, and then run in parallel during the whole trial period. The stepwise introduction of intervention components was designed to promote adherence. All intervention components were standardized, ensuring similar intervention content and intensity for all participants at all study centers, while enabling national adaptations and tailoring to individual needs (for examples see Table 2). Social stimulation was integrated into the different intervention domains through group activities that include high levels of social interactions.

\section{The nutritional intervention}

The nutritional intervention was delivered through 3-4 group sessions (about 60-75 min each) and 3 individual face-to-face counselling sessions (about $30 \mathrm{~min}$ each) with the study dietitian. The individual counselling sessions included careful assessment of the participant's current dietary habits and provided tailored, concrete advice on how to improve diet and implement the changes in their daily routines. The group sessions provided more information, motivation and resources to facilitate lifestyle changes, practical educational activities, while additional support was offered through written information, e.g. tips on food choices, recipes, FAQs. Food frequency questionnaires and 3-day food diaries were administered to monitor diet and dietary changes. Study partners were invited and encouraged to participate in both the individual and group sessions. Participants were advised to consume a diet with the following dietary goals in nutrient intake level that are consistent with New Nordic Recommendations (NNR) 2012 (22) or adapted to the country's nutritional recommendations (i.e., France and Germany). Participants were advised to consume protein $15-20 \mathrm{E} \%$; total fat $25-40 \mathrm{E} \%$, of which saturated fatty acids $<10 \mathrm{E} \%$ and trans-fatty acids as low as possible; monounsaturated fatty acids 10-20 E\%; Polyunsaturated fatty acids 5-10 E\% of which n-3 $>1$ E\%; carbohydrates 45-60 E\%, of which refined sugar $<10 \mathrm{E} \%$; dietary fibre 25-35 g/day; salt $(\mathrm{NaCl}) \leq 6 \mathrm{~g} /$ day; alcohol $\leq 5 \mathrm{E} \%$. These dietary goals are achieved by advising participants to: change from butter and other saturated fats to vegetable fats; consume rapeseed oil, olive oil and vegetable margarines ( $\geq 60 \%$ fat) $\geq 20 \mathrm{~g} /$ day; consume fruit and vegetables according to the recommendation $(\geq 500 \mathrm{~g} /$ day), choose whole grain in all cereal products; limit sucrose intake to $\leq 50 \mathrm{~g} /$ day; consume alcoholic beverages at a maximum of 2 units/day for men and 1 unit/day for women. Recommendations were adjusted according to individual needs related to comorbid conditions, medications and body weight. Vitamin D supplementation (10-20 $\mu \mathrm{g} /$ day) (23) was recommended according to each country's recommendations.

\section{The physical exercise training program}

The physical exercise program was based on international guidelines $(24,25)$ and represented a modified version of the FINGER program (6) and the 
Table 3. Description of the MIND-ADMINI exercise program

\begin{tabular}{|l|l|l|}
\hline Average & $\begin{array}{l}0-3 \text { mo. (Design: } 4 \text { x three weeks bouts to } \\
\text { accustom to exercising) }\end{array}$ & $4-6$ mo. \\
\hline Resistance exercise & $\begin{array}{l}\text { Group training 4-5 participants / PT or } \\
\text { individual exercise }\end{array}$ & $\begin{array}{l}\text { Group training 4-5 participants / PT or } \\
\text { individual exercise }\end{array}$ \\
\hline $\begin{array}{l}\text { Exercise frequency/w } \\
\text { Duration of exercise/minutes }\end{array}$ & 2 & 2 \\
\hline $\begin{array}{l}\text { Number of muscle groups } \\
\text { Repetition/ set }\end{array}$ & 60 & 60 \\
\hline Load \%1 RM * & $8-8$ (primarily legs / core muscles) & $8-10$ \\
\hline Number of sets & Bout 1:.40-50 & $10-20$ \\
\hline $\begin{array}{l}\text { Aerobic exercise } \\
\text { Group training 4-5 participants / PT }\end{array}$ & Bout 3: $60-80$ & $60-80$ \\
\hline
\end{tabular}

${ }^{*} \mathrm{RM}=$ Repetition Maximum. $1 \mathrm{RM}$ corresponds to the highest load that can be lifted through the entire range of motion once

ADEX trial (26). Training was organized in small group sessions which were supervised by a physiotherapist or a personal trainer, preferably $4-5$ participants/trainer (26). The exercise program was tailored to participants' individual physical fitness level and included cardiovascular endurance training and progressive strength training twice a week (about 60 min per session). To facilitate adapting to the exercise program it followed a 3-week bouts design (see Table 3), alternating with resistance exercise and aerobic exercise, both with increasing intensity during the first 12 weeks. During the remaining weeks of the trial, exercise sessions followed a circuit training program alternating strength and aerobic exercises. The strength training program was based on 1 repetition maximum (1RM) and was assessed at baseline and 3 months (additionally at 6 months in the extension study in Sweden), for four main muscle groups (legs, abdomen, back and arms), with a focus on legs and core muscles. Aerobic exercise at the gym was adjusted with the help of the cycle ergometer test results (27) and the Borg scale (28). This was used to ensure that the perceived intensity remains within the right range (not too intense nor too light). Individual aerobic training, such as Nordic walking, cycling and swimming, was encouraged, and all participants were offered advice on how to increase physical activity levels in their daily lives. Additional exercise support was offered through oral and/or written information, e.g. tips on overcoming motivational factors. Participants were monitored for physical activity levels by an accelerometer for one week at baseline, 3 months and 6 months (12 months in the extension study in Sweden) to monitor participants' progress.

\section{Cognitive training}

Cognitive training included group and individual sessions. The 2-3 group sessions (duration approximately 60-75 min per session) were led by psychologists or occupational therapists and involved: general information about neurocognitive disorders, coping and reasoning strategies, introducing the cognitive training program and instructing its use. An additional group session was added in the 6-months extension (in Sweden). Individual training sessions consisted of computer-based training at home or together with other participants, each with their own computer, at the study site (twice a week, approximately 15-30 min per session). The cognitive training program is a web-based, in-house developed computer program including several tasks adapted from protocols previously used in the FINGER trial (6). The program consisted of eight tasks and targeted four cognitive functions: executive processes (updating spatial locations, updating numbers, and a mental setshifting task), working memory (a spatial maintenance task), episodic memory (relational and spatial memory tasks), and mental speed (odd-even and high-low number tasks). As in the original FINGER trial, the program automatically adjusted the level of task difficulty for all tasks (except the shifting task) to the individual performance, by a requirement of $80 \%$ success rate for the next difficult level. The tasks were arranged in two blocks (A and B) and were practiced every other session. This program was adapted in MIND-ADmini by the addition of a less demanding entry level in the updating tasks. The participants were encouraged to perform the cognitive training program at home, and they were provided with individual log-in accounts and instructions for using the web-based program. They were also encouraged to contact the psychologist to receive any support they needed to manage their training from home.

\section{Social stimulation}

Social activities were stimulated through the numerous group meetings within the intervention domains (physical exercise, diet, and cognitive training). The group sessions were designed to facilitate open discussions 
and interactions with other participants. One example is the physical exercise sessions which were performed twice a week in the same small groups throughout the intervention period. After each training exercise session, participants were offered a healthy snack and had the opportunity to freely discuss any aspects related to physical activity with each other and the trainer. Furthermore, study partners were encouraged to participate in all sessions.

\section{Management of metabolic and vascular risk factors}

Management of metabolic and vascular risk factors was based on national evidence-based guidelines (29). It included one additional meeting with the study nurse (at 3 months), for measurements of blood pressure, weight and BMI, hip and waist circumference, and further recommendations for lifestyle management and to enhance motivation. If medication initiation or adjustments were needed, the study physician either wrote a prescription or referred the participant to regular healthcare, as appropriate according to local procedures. Smokers were also provided with support to quit smoking.

\section{The medical food intervention}

The lifestyle + medical food intervention group received all the above-mentioned lifestyle intervention components and in addition the study product Fortasyn Connect, a $125 \mathrm{ml}$ once-a-day milk-based drink. The specific multi-nutrient combination Fortasyn Connect contains long-chain omega-3 fatty acids docosahexaenoic acid (DHA) and eicosapentaenoic acid (EPA), uridine monophosphate, choline, vitamins B12, B6, C, E, and folic acid, phospholipids, and selenium. This combination of nutrients was designed to enhance efficacy over what can be achieved by individual nutrients. For example, it synergistically enhances the formation of neuronal membranes by providing rate limiting compounds for the synthesis of brain phospholipids, and targets AD-related pathological processes. In Sweden, Finland, and Germany Fortasyn Connect (Souvenaid ${ }^{\mathrm{TM}}$ ) is available on the market in $125 \mathrm{ml}$ bottles. Danone Nutricia Research provided this product for the MIND-ADmini trial.

\section{Follow-up and outcome measurements}

All participants (control and intervention groups) met the study nurse at screening, baseline, and at 6 months (additionally at 12 months in the extension study in Sweden) for measurements of blood pressure, weight and BMI, and hip and waist circumference. All participants (control and intervention groups) met the study physician at screening and at 6 months for a detailed medical history and physical examination. At baseline and at 6 months, the cognitive status of the participants was assessed by a psychologist, and information on health status, lifestyle, demographic and socioeconomic factors was collected.

The primary outcome of the MIND-ADmini pilot trial is feasibility of the multimodal intervention measured by:

(1) Recruitment rate defined as proportion of participants who are randomized of those who fulfilled the criteria and were invited to participate during a 6-month recruitment phase. We consider a recruitment rate of $50 \%$ or more as successful.

(2) Overall adherence to the intervention measured in each of the two intervention arms (composite measure of participation in different intervention components). Several measures of adherence will be used: for cognitive training, attendance at the cognitive training sessions including automatic recordings of computer program use, and group sessions attendance; for physical exercise, participation in exercise sessions; for nutrition, attendance at the group and individual nutrition sessions; for vascular care, attendance at the 3-month follow-up visit with the study nurse, where measurements are taken for blood pressure, weight, BMI, hip and waist circumference; for medical food, consumption of medical food product measured through a study product diary, similar to LipiDiDiet trial. If a participant attends a minimum of $40 \%$ of sessions per domain, in at least $2 / 4$ domains (exercise, nutrition, cognitive training and monitoring of vascular/metabolic risk factors), the participant is considered to successfully adhere to the lifestyle intervention. In the lifestyle + medical food intervention arm, the participant is considered to successfully adhere to the intervention if they in addition consume at least $60 \%$ of the medical food study product.

(3) Retention rate defined as proportion of participants who complete the 6-month trial period. We consider a successful rate if less than $35 \%$ of participants drop out. The reason for drop-out will be recorded as provided by the participant.

Secondary outcomes are:

(1) Adherence to intervention components in each of the two intervention arms i.e. adherence to exercise (attendance at the exercise sessions), nutritional guidance (attendance at the nutrition sessions), cognitive training (including attendance at the group sessions and cognitive training sessions), intensive monitoring of vascular/ metabolic risk factors (session attendance), and medical food adherence (study product diary similar to LipiDiDiet trial).

(2) Adherence to healthy lifestyle changes, monitored with the composite healthy lifestyle score, which will be based on several components and calculated for all trial participants. The healthy lifestyle score will be based on several components. For the exercise component, short physical activity questionnaire (frequency and duration of activity, Swedish National Board of Health and Welfare (30)) will be used, and objective measurements (e.g. actigraph) will also be considered. For nutritional guidance, the HATICE food questionnaire (measure from 
Table 4. Preliminary baseline characteristics of the trial participant

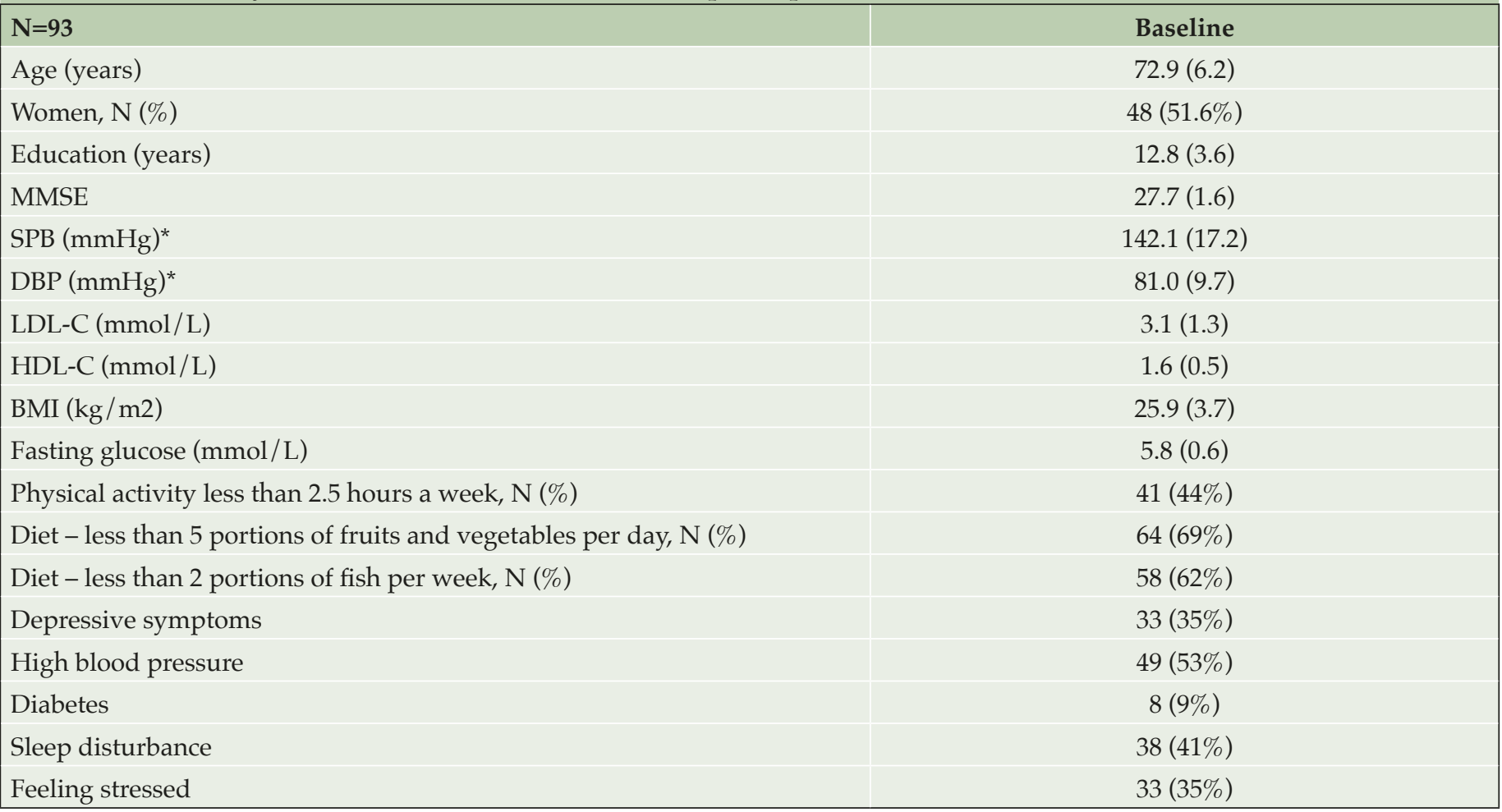

* Data not available for 26 participants. Characteristics are calculated based on number of participants with available data. Numbers are means (SD) unless otherwise specified.

the European diabetes projects ePredice trial) is used. Cognitive activities are measured through questions regarding participation/engagement in cognitively stimulating activities. For vascular/metabolic risk factors, adherence to national guidelines is assessed. Measures used in the original FINGER trial will also be considered.

Exploratory outcomes are: changes in vascular and metabolic risk factors; depressive symptoms (Geriatric Depression Scale) (31); stress-related symptoms (Perceived Stress Scale) (32); physical performance; timed 10-meter walking test; timed 10-meter dual-task test (33); 30 second chair stand test and Short Physical Performance Battery (SPPB) (34); health-related quality of life (RAND36) (35); Insomnia Severity Index (36); blood biomarkers (e.g. lipids, markers for inflammation, vitamins D \& B); experiences of the participants (qualitative data based on interviews).

Cognition was measured through a detailed cognitive assessment (Neuropsychological Test Battery, NTB) and functional assessments (Clinical Dementia Rating, CDR (37) and CDR sum of boxes; and Alzheimer's Disease Cooperative Study-Activities of Daily Living, ADCS-ADL (38)) at baseline and at the 6-month visit to obtain reliable estimates of change over time for power calculations for a future larger multimodal intervention trial based on the current pilot trial.

\section{Statistical considerations}

The MIND-ADmini trial was expected to include up to 150 participants. The recent CONSORT extension guidelines for developing and reporting pilot RCTs state that "no hypothesis testing is recommended" for pilot trials (39). No formal sample size calculations were needed because the primary outcome measures are feasibility (recruitment and intervention acceptability i.e., adherence and retention), safety and adherence to the multimodal intervention in participants with prodromal AD. All analyses will be conducted at the group level. The three trial arms will be compared to assess the differences in the primary, secondary and exploratory outcomes.

\section{Ethics and safety aspects}

MIND-ADmini was approved by local ethical committees in all four countries. Participants and study partners gave their written informed consent prior to enrollment in the trial. Additional informed consent was obtained before participation in the optional 6-month extension study. The trial adhered to the Declaration of Helsinki and is conducted according to the International Conference on Harmonisation (ICH) guidelines for Good Clinical Practice (GCP). Study participants were insured according to local legislation. Participants had the possibility to contact the study staff throughout the study and they were referred to appropriate medical care 
when indicated. The lifestyle intervention protocol was adjusted for the prodromal AD target group. The safety of the intervention was assessed, and data were collected on adverse events (AEs) and serious adverse events (SAEs).

Data management process: A logistic system to schedule appointments was created and designed by the MIND-AD Consortium. Form templates and data collection files were electronically provided by the trial coordinators, and study forms were printed at each center. For validated scales, already available translated versions were used. Other documents were translated at the local trial centers. Source data and other data collection forms were entered in an electronic case report form (eCRF) using the SMART-TRIAL platform (40). Monitors verified the completed CRFs against the source data and trial database. The Investigator Site File was regularly updated according to ICH-GCP guidelines.

\section{Study progress}

The study started in September 2017 in Sweden, November 2017 in Finland, April 2018 in Germany and October 2018 in France. In total, 93 participants were randomized, and the 6-month intervention was completed in December 2019. Final data checks and database lock were delayed due to Covid-19 pandemic restrictions. Data analysis is ongoing.

Baseline characteristics of the participants are summarized in Table 4. The mean (SD) age of the entire sample was 72.9 years, level of education was 12.8 years, and MMSE score 27.7 points. Vascular and lifestylerelated risk factors were common as expected, based on the inclusion criteria: Impaired fasting glucose $(\geq 6.1$ $\mathrm{mmol} / \mathrm{L}$ ) was seen in $21 \%$, and $57 \%$ were overweight, with $\mathrm{BMI} \geq 25$ and, $13 \%$ had $\mathrm{BMI} \geq 30$. The baseline characteristics of participants are presented in Table 4 .

\section{Discussion}

The MIND-ADmini trial investigates whether a multimodal lifestyle-based intervention is feasible among individuals with prodromal AD in four European countries (12). Finding effective and feasible prevention strategies is particularly important for individuals with prodromal AD who currently lack effective treatments. While multimodal lifestyle interventions have been indicated to be efficient and feasible among individuals at risk for dementia, their feasibility and effects have not been studied in RCT settings among individuals who already have cognitive impairment. Knowledge is needed about the required dosage and frequency of the multimodal intervention and whether adaptations of the intervention components (e.g. nutrition, exercise, cognitive training) can ensure adherence and optimize the benefits.

Multidomain RCTs for dementia prevention and risk reduction have been adopting novel designs and implementation strategies. The MAPT trial for example combined omega 3 polyunsaturated fatty acid supplementation with a multimodal lifestyle intervention (nutritional advice, physical activity and cognitive training), and tested their effects alone or in combination among older adults with memory complaints (9). Similarly, the MIND-ADmini tested a multimodal lifestyle intervention alone or combined with medical food (Fortasyn Connect). This is an important step towards combining pharmacological and non-pharmacological interventions. The MIND-ADmini trial builds on previous experiences from multimodal prevention trials, and incorporates recent suggestions for prevention interventions to target at-risk individuals, as opposed to non-selected groups (41). Further, MIND-ADmini will contribute to important methodological advancements in prevention trials, as it will collect feedback on participants' experiences through qualitative interviews. Experiences from MIND-ADmini will aid in designing larger efficacy trials, as well as future large international multimodal trials among at-risk populations.

In current $\mathrm{AD}$ research, there is an increasing emphasis on early interventions, e.g. for individuals in an earlysymptomatic disease stage such as prodromal AD. In MIND-ADmini the IWG-1 criteria for prodromal AD (12) were used, even though prodromal AD can now be defined using newer research diagnostic criteria (11, 42). One reason for using the IWG-1 criteria is that they are more pragmatic and easier to apply in multinational settings. This is mainly because there is more flexibility in terms of which biomarkers can be tested, and CSF is not required. Also, these criteria were used in the LipiDiDiet trial $(13,14)$. Using these IWG-1 criteria in LipiDiDiet, $88 \%$ of participants (with centrally analyzed CSF available) were confirmed to be amyloid positive, meaning that these criteria performed well at identifying a prodromal AD population with underlying amyloid pathology (43).

To further advance multidomain lifestyle intervention trials for dementia risk reduction, the World-Wide FINGERS (WW-FINGERS) network was launched in 2017 (44). WW-FINGERS is the first global network of multidomain lifestyle intervention trials for dementia risk reduction and prevention, including over 35 countries in different geographical, cultural and economic settings (45). WW-FINGERS aims to harmonize and adapt multidomain interventions across various countries and settings, facilitate data sharing and analyses across studies, and promote international joint initiatives, to identify globally implementable and effective preventive strategies. WW-FINGERS will also create a unique opportunity for rapid knowledge dissemination and implementation, and the MIND-ADmini trial will contribute to this as a valuable resource since it serves as a model for combination therapy trials (non-pharma, nutrition-based and/or pharmacological interventions).

Acknowledgements: This is an EU Joint Programme - Neurodegenerative Disease Research (JPND) project. The project is supported through the following funding 
organisations under the aegis of JPND - www.jpnd.eu: Finland, Academy of Finland; France, L'Agence Nationale de la Recherche (The French National Research Agency, ANR-14-JPPS-0001-02); Germany, Bundesministerium für Bildung und Forschung (BMBF) (The Federal Ministry of Education and Research, FKZ01ED1509 and 01ED2003); Sweden, Swedish Research Council), The Netherlands, ZonMw (The Netherlands Organisation for Health Research and Development, 733051041). The project is additionally supported by Alzheimerfonden, Center for Innovative Medicine (CIMED) at Karolinska Institutet South Campus, Hjärnfonden, the Knut and Alice Wallenberg Foundation, and the Konung Gustaf V:s och Drottning Victorias Frimurarestiftelse. Join Program of Neurodegenerative Disorders - prevention (EURO-FINGERS). Region Stockholm (ALF, NSV), Stiftelsen Stockholms sjukhem, Swedish Research Council for Health Working Life and Welfare (FORTE), European Research Council grant 804371. The authors would also like to thank all members of the MIND-AD Consortium.

\section{Trial registration: ClinicalTrials.gov NCT03249688}

Conflict of Interest: Dr. Andrieu reports grants from EU - JPND program MIND-AD project, during the conduct of the study; grants from EU - H2020 program - PRODEMOS project, grants from CNAV, personal fees and nonfinancial support from Nestlé SA, outside the submitted work. Dr. Broersen is employee of Danone Nutricia Research. Dr. Coley reports grants from Agence Nationale de la Recherche (ANR), during the conduct of the study. Dr. Wimo reports personal fees from Biogen, from Eisai, grants from Merck, outside the submitted work. Dr. Soininen reports grants from Academy of Finland for JNPD EU project, during the conduct of the study; personal fees from Consultation fee, outside the submitted work. Dr. Hartmann reports grants from BMBFJPND, grants from EU FP7 LipiDiDiet, during the conduct of the study. Dr. Solomon reports grants from European Research Council grant 804371, grants from Alzheimerfonden, grants from Academy of Finland, during the conduct of the study. Dr. Kivipelto reports grants from The EU Joint Programme Neurodegenerative Disease Research (JPND), Alzheimerfonden, Hjärnfonden, Center for Innovative Medicine (CIMED) at Karolinska Institutet South Campus, Knut and Alice Wallenberg Foundation, Konung Gustaf V:s och Drottning Victorias Frimurarestiftelse, Joint Program of Neurodegenerative Disorders - prevention (EURO-FINGERS), Region Stockholm (ALF, NSV), Stiftelsen Stockholms sjukhem, Swedish Research Council for Health Working Life and Welfare (FORTE), during the conduct of the study; and was an invited speaker and received an honorarium at an industry-led symposium including Biogen, Nutricia and Nestlé, outside the submitted work.

Open Access: This article is distributed under the terms of the Creative Commons Attribution 4.0 International License (http:/ / creativecommons.org/ licenses/by/4.0/), which permits use, duplication, adaptation, distribution and reproduction in any medium or format, as long as you give appropriate credit to the original author(s) and the source, provide a link to the Creative Commons license and indicate if changes were made.

\section{References}

1. Livingston, G., et al., Dementia prevention, intervention, and care: 2020 report of the Lancet Commission. Lancet, 2020. 396(10248): p. 413-446 DOI: 10.1016/ S0140-6736(20)30367-6.

2. World Health Organisation. Risk Reduction of Cognitive Decline and Dementia: WHO Guidelines. 2019 [cited 201920 December]; Available from: https: / / www.ncbi.nlm.nih.gov/pubmed/31219687.

3. Solomon, A., et al., Advances in the prevention of Alzheimer's disease and dementia. J Intern Med, 2014. 275(3): p. 229-50 DOI: 10.1111/joim.12178.

4. Boyle, P.A., et al., Attributable risk of Alzheimer's dementia attributed to agerelated neuropathologies. Ann Neurol, 2019. 85(1): p. 114-124 DOI: 10.1002/ ana.25380.

5. Kivipelto, M., F. Mangialasche, and T. Ngandu, Lifestyle interventions to prevent cognitive impairment, dementia and Alzheimer disease. Nat Rev Neurol, 2018. 14(11): p. 653-666 DOI: 10.1038/s41582-018-0070-3.

6. Ngandu, T., et al., A 2 year multidomain intervention of diet, exercise, cognitive training, and vascular risk monitoring versus control to prevent cognitive decline in at-risk elderly people (FINGER): a randomised controlled trial. Lancet, 2015. 385(9984): p. 2255-63 DOI: 10.1016/S0140-6736(15)60461-5.

7. Kivipelto, M., et al., Risk score for the prediction of dementia risk in 20 years among middle aged people: a longitudinal, population-based study. The Lancet Neurology, 2006. 5(9): p. 735-741 DOI: 10.1016/S1474-4422(06)70537-3.

8. Moll van Charante, E.P., et al., Effectiveness of a 6-year multidomain vascular care intervention to prevent dementia (preDIVA): a cluster-randomised controlled trial. Lancet, 2016. 388(10046): p. 797-805 DOI: 10.1016/S01406736(16)30950-3.

9. Andrieu, S., et al., Effect of long-term omega 3 polyunsaturated fatty acid supplementation with or without multidomain intervention on cognitive function in elderly adults with memory complaints (MAPT): a randomised, placebo-controlled trial. Lancet Neurol, 2017. 16(5): p. 377-389 DOI: 10.1016/
S1474-4422(17)30040-6.

10. Kivipelto, M., Mangialasche, F., Ngandu, T, Can lifestyle changes prevent cognitive impairment? Lancet Neurol, 2017. DOI 10.1016/S14744422(17)30080-7.

11. Dubois, B., et al., Advancing research diagnostic criteria for Alzheimer's disease: the IWG-2 criteria. Lancet Neurol, 2014. 13(6): p. 614-29 DOI: 10.1016/ S1474-4422(14)70090-0.

12. Dubois, B., et al., Research criteria for the diagnosis of Alzheimer's disease: revising the NINCDS-ADRDA criteria. Lancet Neurol, 2007. 6(8): p. 734-46 DOI: 10.1016/S1474-4422(07)70178-3.

13. Soininen, $\mathrm{H}$., et al., 24-month intervention with a specific multinutrient in people with prodromal Alzheimer's disease (LipiDiDiet): a randomised, double-blind, controlled trial. Lancet Neurol, 2017. 16(12): p. 965-975 DOI: 10.1016/S1474-4422(17)30332-0.

14. Soininen, $\mathrm{H}$., et al., 36-month LipiDiDiet multinutrient clinical trial in prodromal Alzheimer's disease. Alzheimers Dement, 2021. 17(1): p. 29-40 DOI: 10.1002/alz.12172.

15. Harbaugh, M.P., et al., Long-chain, n-3 fatty acids and physical activity-independent and interactive associations with cardiac autonomic control. Int J Cardiol, 2013. 167(5): p. 2102-7 DOI: 10.1016/j.ijcard.2012.05.110.

16. Muldoon, M.F., et al., Concurrent physical activity modifies the association between $\mathrm{n} 3$ long-chain fatty acids and cardiometabolic risk in midlife adults. J Nutr, 2013. 143(9): p. 1414-20 DOI: $10.3945 /$ jn.113.174078.

17. Pastor, R. and J.A. Tur, Response to exercise in older adults who take supplements of antioxidants and/or omega-3 polyunsaturated fatty acids: A systematic review. Biochem Pharmacol, 2020. 173: p. 113649 DOI: 10.1016/j. bcp.2019.113649.

18. Kaufman, C.S., et al., Self-Reported Omega-3 Supplement Use Moderates the Association between Age and Exercising Cerebral Blood Flow Velocity in Older Adults. Nutrients, 2020. 12(3) DOI: 10.3390/nu12030697.

19. Chhetri, JK, et al, Effects of a 3-Year Multi-Domain Intervention with or without Omega-3 Supplementation on Cognitive Functions in Older Subjects with Increased CAIDE Dementia Scores. J Alzheimers Dis, 2018. 64(1): p. 71-78 DOI: $10.3233 /$ JAD-180209.

20. Caldwell, C.C., J. Yao, and R.D. Brinton, Targeting the Prodromal Stage of Alzheimer's Disease: Bioenergetic and Mitochondrial Opportunities. Neurotherapeutics, 2015. 12(1): p. 66-80 DOI: 10.1007/ s13311-014-0324-8.

21. Folstein, M.F., S.E. Folstein, and P.R. McHugh, "Mini-mental state". A practical method for grading the cognitive state of patients for the clinician. J Psychiatr Res, 1975. 12(3): p. 189-98 DOI: 10.1016/0022-3956(75)90026-6.

22. Swedish National Food Agency. Nordic nutrition recommendations. 2012 [cited 201927 April]; Available from: https://www.livsmedelsverket. se/en/food-habits-health-and-environment/dietary-guidelines / naringsrekommendationer.

23. Vieth, R., Vitamin D supplementation, 25-hydroxyvitamin D concentrations, and safety. The American Journal of Clinical Nutrition, 1999. 69(5): p. 842-856 DOI: $10.1093 /$ ajcn/69.5.842

24. Nelson, M.E., et al., Physical activity and public health in older adults: recommendation from the American College of Sports Medicine and the American Heart Association. Med Sci Sports Exerc, 2007. 39(8): p. 1435-45 DOI: $10.1249 /$ mss.0b013e3180616aa2.

25. World Health Organisation. Global action plan on physical activity 2018-2030: more active people for a healthier world. 20181 June 2018 [cited 202012 January]; Available from: https://www.who.int/publications-detail/globalaction-plan-on-physical-activity-2018\%E2\%80\%932030.

26. Hoffmann, K., et al., Preserving Cognition, Quality of Life, Physical Health and Functional Ability in Alzheimer's Disease: The Effect of Physical Exercise (ADEX Trial): Rationale and Design. Neuroepidemiology, 2013. 41(3-4): p. 198207 DOI: $10.1159 / 000354632$.

27. Dahl, H.A., et al., Textbook of work physiology : physiological bases of exercise. 4 ed. 2003, Champaign Human kinetics. 649.

28. Borg, G., P. Hassmén, and M. Lagerström, Perceived exertion related to heart rate and blood lactate during arm and leg exercise. European Journal of Applied Physiology and Occupational Physiology, 1987. 56(6): p. 679-685 DOI: 10.1007/BF00424810

29. Piepoli, M.F., et al., 2016 European Guidelines on cardiovascular disease prevention in clinical practice: The Sixth Joint Task Force of the European Society of Cardiology and Other Societies on Cardiovascular Disease Prevention in Clinical Practice (constituted by representatives of 10 societies and by invited experts)Developed with the special contribution of the European Association for Cardiovascular Prevention \& Rehabilitation (EACPR). European heart journal, 2016. 37(29): p. 2315-2381 DOI: 10.1093 eurheartj/ehw106.

30. Swedish National Board of Health and Welfare. Swedish National Guidelines on prevention and lifestyle habits 2011 [cited 201721 jan].

31. Yesavage, J.A., Geriatric Depression Scale. Psychopharmacol Bull, 1988. 24(4): p. 709-11.

32. Cohen, S., T. Kamarck, and R. Mermelstein, A global measure of perceived stress. J Health Soc Behav, 1983. 24(4): p. 385-96. 
33. Hunter, S.W., et al., A framework for secondary cognitive and motor tasks in dual-task gait testing in people with mild cognitive impairment. BMC geriatrics, 2018. 18(1): p. 202-202 DOI: 10.1186/s12877-018-0894-0.

34. Guralnik, J.M., et al., A short physical performance battery assessing lower extremity function: association with self-reported disability and prediction of mortality and nursing home admission. J Gerontol, 1994. 49(2): p. M85-94 DOI: 10.1093/geronj/49.2.m85.

35. Hays, R.D., C.D. Sherbourne, and R.M. Mazel, The RAND 36-Item Health Survey 1.0. Health Econ, 1993. 2(3): p. 217-27 DOI: 10.1002/ hec.4730020305.

36. Morin, C.M., Insomnia: Psychological assessment and management. Insomnia: Psychological assessment and management. 1993, New York, NY, US: Guilford Press. xvii, 238-xvii, 238.

37. Berg, L., Clinical Dementia Rating (CDR). Psychopharmacol Bull, 1988. 24(4): p. 637-9.

38. Galasko, D., et al., An inventory to assess activities of daily living for clinical trials in Alzheimer's disease. The Alzheimer's Disease Cooperative Study. Alzheimer Dis Assoc Disord, 1997. 11 Suppl 2: p. S33-9.

39. Thabane, L., Hopewell, S., Lancaster, G. A., Bond, C. M., Coleman, C. L., Campbell, M. J., \& Eldridge, S. M, Methods and processes for development of a CONSORT extension for reporting pilot randomized controlled trials. Pilot and Feasibility Studies, 2016. 2(1): p. 1. DOI: 10.1186/s40814-016-0065-z.
40. SMART-trial. SMART-trial. [Electronic case report form] 202124 Febeuary 2021 [cited 20215 January]; Available from: https: / / www.smart-trial.com/ecrf.

41. Schneider, L.S., Reduce vascular risk to prevent dementia? Lancet, 2016 388(10046): p. 738-40 DOI: 10.1016/S0140-6736(16)31129-1.

42. Jack, C.R., Jr., et al., NIA-AA Research Framework: Toward a biological definition of Alzheimer's disease. Alzheimer's \& dementia : the journal of the Alzheimer's Association, 2018. 14(4): p. 535-562 DOI: 10.1016/j.jalz.2018.02.018.

43. Rosenberg, A., et al., Research diagnostic criteria for Alzheimer's disease: findings from the LipiDiDiet randomized controlled trial. Alzheimers Res Ther, 2021. 13(1): p. 64 DOI: 10.1186/s13195-021-00799-3.

44. Kivipelto, M., et al., World Wide Fingers will advance dementia prevention. Lancet Neurol, 2018. 17(1): p. 27 DOI: 10.1016/S1474-4422(17)30431-3.

45. Kivipelto, M., et al., World-Wide FINGERS Network: A global approach to risk reduction and prevention of dementia. Alzheimers Dement, 2020. 16(7): p. 1078-1094 DOI: 10.1002 / alz.12123.

How to cite this article: S. Sindi, C. Thunborg, A. Rosenberg, et al. Multimodal Preventive Trial for Alzheimer's Disease: MIND-ADmini Pilot Trial Study Design and Progress. J Prev Alz Dis 2022;1(9):30-39, http: / / dx.doi.org/10.14283/ jpad.2022.4 